The next four chapters report the “... more obvious, most sensational and/or the most frequently described findings following frontal lobe damage." These include disorders of motor function, attention, awareness, personality and emotion. Aphasia, despite its obvious and classical association with frontal lobe pathology is not discussed at this point, but is reserved for more detailed discussion later in the text. Throughout the book, the authors point out that the frontal lobes cannot be regarded as an autonomous and homogeneous anatomical entity, serving any single, obvious function. Rather, damage to the frontal cortex seems to have an influence on almost all aspects of cognitive behavioural function, forcing us to break down rigid anteriorposterior distinctions of brain organisation. This integrative approach is emphasised in the chapters on sensory-perceptual function, language and speech, memory, cognition and executive functions. The authors argue further that many of the neuropsychological instruments which we have available to us are unsuitable for analysing the true nature of frontal lobe function. They emphasise the need for more controlled, experimental procedures, an approach which would be of benefit to neuropsychology as a whole.

The final three chapters attempt to tie up some loose ends. Chapter 15 considers what we can and cannot say about localisation of function in the frontal lobes, while Chapter 16 reviews some of the major theories of frontal lobe function, both historical and contemporary. This latter chapter might have been usefully placed early in the text. The theoretical context within which studies were undertaken is of help in appreciating the diverse literature. Placed at the end, the theory seems little more than an afterthought. More validly, the final chapter is the author's own attempt to integrate the mass of accumulated behavioural and anatomical evidence, ranging in the process, from attention to free will. The attempt is, as the authors recognise, premature and incomplete. It is also, inevitably, disquietingly vague. It is clear that we have become locked into an approach to neuropsychology which demands clearly definable symptoms on which to base our theories. The book illustrates that we have yet to clearly define those symptoms when it comes to the frontal lobes. We also need a new language of neuropsychology to allow us to redefine existing evidence, and derive future theory. This book attempts to gather up a vast and amorphous body of knowledge. Hopefully the exercise will serve to stimulate a new direction for future research.

RG BROWN
Neurosonography of the Pre-Term Neonate. Edited by Edward G Grant. (Pp 116; DM 138.00.) Heidelberg: Springer-Verlag, 1986.

This book will be of interest to everybody who is involved in cranial ultrasonography in the neonatal intensive care unit.

Although it is not a comprehensive book of cranial ultrasonography, it deals in detail with germinal matrix related haemorrhages and periventricular leukomalacia in the premature infant.

The authors start with a chapter about the normal anatomy. This is very well illustrated and the legends are very clear. Subsequently they give us some insight in the pathogenesis of haemorrhages and leukomalacia. The third chapter covers the different sizes of germinal matrix related haemorrhages (GMRH). A lot of useful information is given to enable one to distinguish a germinal layer from an intraventricular haemorrhage. The fourth chapter deals with periventricular leukomalacia and contains many beautiful illustrations. The next two chapters look at the outcome of the survivors with haemorrhages and those with leukomalacia. The last chapter deals with the comparison of two modalities, ultrasound and computed tomography.

All the illustrations in this book are of very good quality. As only the areas of germinal matrix related haemorrhages and leukomalacia are dealt with, other books will still be required to cover other important areas, such as the different types of infections and the congenital abnormalities. As these problems are also encountered in the premature infant, it is difficult to understand why these matters are not included in this book.

Little information is given about the different methods of measuring the size of the ventricles. The authors are apparently not very keen on performing any measurements. Measurements, performed once or twice a week may be very helpful in choosing the right time to intervene when ventricular dilatation appears to be progressive.

The authors stress very well the pitfalls in diagnosing leukomalacia and show interesting follow-up scans, such as ventricular dilatation and widening of the interhemispheric fissure, the latter not having been reported previously.

It is nice to find an imaging book which contained two chapters dealing with the outcome of the infants with the different types of brain lesions. The number of infants available for follow-up in the haemorrhage group is a bit disappointing, but the data available do support findings in other follow-up studies. The number of infants with normal ultrasound scans who subsequently developed major or minor handicaps was quite high. This could be due to the fact that transient periventricular densities have too often been diagnosed as a normal periventricular halo. More evidence has recently become available that these transient periventricular densities, also called "flares" are the milder end of the spectrum of leukomalacia. The follow-up data of the infants with cystic leukomalacia are more complete and show the poor prognosis of the survivors.

The last chapter which compares ultrasound with CT is a useful guide for those involved in the management of these infants and will help them to choose the right imaging technique for diagnosing the different types of lesion. They show that nonhaemorrhagic leukomalacia, or strands or septi in the ventricles can be missed using CT scans, but that convexity lesions are more readily seen using this technique.

This book can be recommended to all involved in scanning premature infants in the neonatal intensive care unit.

LINDA DE VRIES

TRH and Spinocerebellar Degeneration. Edited by Itsuro Sobue. (Pp 268; \$74.00.) Amsterdam: Elsevier Press, 1986.

The attempts to identify an effective therapy for ataxia which have been made in the last decade or so do not add up to a tremendous success story. Impressive results from one centre, regrettably infrequently obtained from a well designed double blind trial, have rarely been confirmed in another.

The rationale for many of the proposed therapies for ataxia, particularly cholinergic compounds, is rather tenuous. Superficially it is difficult to see why thyrotropin releasing hormone (TRH) should confer any benefit on ataxic patients. TRH was first shown to improve ataxia in an ataxic mouse mutant (rolling mouse Nagoya) in 1977. Noradrenaline is present in abnormally high concentrations in the cerebellum and brainstem of these mice, and TRH accelerates noradrenaline turnover in the brain. It is known that there are noradrenergic afferents projecting to the mammalian cerebellar cortex, although their function is unclear. A recent observation which is possibly important, but not stressed in this volume, is that TRH appears to play a role in regulating GABA receptors in the cerebellum; GABA is the major neurotransmitter of Purkinje, basket and Golgi cells.

In this book. Sobue and many con- 
tributors from Japan describe the effects of TRH and TRH analogues, administered parenterally and orally, on patients with a variety of degenerative ataxic disorders. There is an extensive introductory section describing the distribution, metabolism, and function of TRH in the nervous system and elsewhere, two chapters on the pharmacokinetics of TRH, and further chapters on the effects of TRH on various mouse models of ataxia. A useful summary of what is known about neurotransmitters and cerebellar function is provided by Kanazawa. Yoshida and Nakanishi review various clinical and other methods of evaluating disability in ataxic patients. Overall the Japanese experience, based on either open or controlled trials, suggests that administration of TRH or its analogue DN-1417 increases stability of stance and gait and improves dysarthria in patients with degenerative ataxias. This book provides a useful starting point for anyone hoping to confirm these results in further trials.

ANITA HARDING

Biology of Brain Tumour. Edited by MD Walker and DGT Thomas. (Pp 515; £125.25.) Dordrecht: Martinus Nijhoff, 1986.

Just over 100 years ago on the 25 November 1884 at The Hospital for Epilepsy and Paralysis, Rickman Godlee performed the first reported operation on a patient with a glioma. The hospital, which was initially in a house off Marylebone High Street, moved in 1872 to a building in Regents Park, the site of which today is part of the London Zoological Gardens. In 1903 the hospital moved to its present site in Maida Vale, and there is irony and sadness in the prospect of that hospital's closure within a century of its foundation.

To mark this surgical centenary, the Second International Symposium on Biology of Brain Tumour was held at the Zoological Society in October 1984; hence the publication of the Proceedings in late 1986 under the title Biology of Brain Tumour. The seven sections in the book are: Molecular Neurooncology, Modern Neuropathology, Correlates of Brain Tumour Imaging, Surgical Neuro-oncology, The Biology of Radiotherapy, Drug Therapy, and ImmunoNeurobiology. The first contribution in most of the sections is something of a "keynote address", the other papers being more or less related to that subject. A few of the contributions were invited, but the majority were papers submitted.

The objective of the Symposium was to bring together a group of research workers, oncologists and clinicians whose major interest was brain tumours, and to record the current "state of the Art". The first was undoubtedly achieved, and as with all such gatherings, those who attended gained much from personal meetings, and private or open discussion with colleagues.

It is the second objective which is more difficult to assess, as is the rightful place of the publication of conference proceedings in general in the form of a relatively expensive $(£ 125 \cdot 25)$ book. For workers in the neurological sciences who are not involved in the subject, either clinically or in the laboratory, the book would have very limited appeal. Had the historical aspects been given greater weight-and it is surprising that the tantalisingly short piece on the surgical history of Maida Vale Hospital was not expanded and given first place - the book would have had a much wider appeal. Similarly the "keynote" communications which should have set the scene for each section, vary greatly in achieving that goal. With the exception of Molecular Biology of Cancer, and Current Approaches to Chemotherapy, the keynote topics are generally disappointing. Some are an unhappy amalgam of the author's own current research interests and an over-view of the subject, and with the limitation of space, neither aspect is developed satisfactorily. Finally for the "general" reader, the value of the book would have been very greatly enhanced if a chapter had been written after the Symposium by the two editors, in which the threads of the Symposium had been drawn together with some indications of the way forward. Michael Walker and David Thomas, with their own very important and extensive contributions to the subject were in a unique position to do this.

For workers in the field of malignant brain tumour, individual contributions will be of value in their content and bibliography. A few contain new ideas, some controversial and even within this one volume there are conflicting results on the same subject, as with the laser. Inevitably there are criticisms of the book as a collection of papers on original work. Some contributors have published their work more fully elsewhere and interested readers will turn to those other sources. It is doubtful whether some of the contributions would have been accepted by journals which referee critically. Lastly, contributions had to be submitted some months before the Symposium, and therefore it is likely that work in some fields was completed 3 years or more prior to publication. Unfortunately such delays seem inevitable when publication takes this form.
The book is well produced in text afid illustrations but with some errors in page numbering in the table of contents. It ma $\mathrm{ks}$ an important occasion, and its value depend upon the needs of the individual reader.

JOHN GARFIEDD

Society of British Neurological Surgeons. The next meeting will be held at the Institufe of Psychiatry, London, 17-18 September 1987. Particulars can be obtained from Te Secretary, Neuroscience Unit, Walsgraye Hospital, Clifford Bridge Road, Walsgraze, Coventry.

\section{European Neurological Society}

The European Neurological Society (BNSB) has been founded to foster coopergtign between neurologists and those working in related disciplines within Europe. Meetings will be held biennially. The participation of individuals from other areas of the wollds welcomed. The biennial meetings willä $\overrightarrow{\mathrm{EG}}-$ vide instructional courses for neurologistsin training, up-date symposia, and sessions for the presentation of original work, either as platform or poster presentations. The fist meeting will be held in Nice from Jueㅏe 19-22, 1988, when the teaching programfle will include courses on peripheral neurgpathy, neuroimaging, dementia, and neurovirology/neuroimmunology. The will be symposia on physiological antd pathological aspects of cerebral blood flow, neuromediators, and neurogenetics.

Further information about the ENS my be obtained from the Secretary, Professor $G$ Said, Service de Neurologie, Hôpital ge Bicêtre, 94275 Le Kremlin Bicêtre Céde France.

The XXXI International Congress on History of Medicine

This Congress will be held in Bologna August-4 September 1988. Information may be obtained from Secretariat, Catted $F$. di Storia della Medicina, Via Massarenting, 40138 Bologna, Italy. 